\title{
FITC Detection System Controlled by Intelligent Mobile Phone
}

\author{
Cheng Ju${ }^{1}$, Zengri Wang ${ }^{1}$, Zhenwu Gong ${ }^{2}$, Zhanqiang Li ${ }^{1}$ and Yunfang Jia ${ }^{1, ~}$ \\ ${ }^{1}$ College of Electronic Information and Optical Engineering, Nankai University, China \\ ${ }^{2}$ Lanlike Chemical electronic high technology Co., Ltd., Tianjin, China \\ *Corresponding author: jiayf@nankai.edu.cn
}

\begin{abstract}
Keywords: Florescence detection, Photo-electronic detecion, Intelligent mobile phone, Android, Bluetooth.

Abstract. In this paper, a specific florescence detection system for florescence isothiocyanate (FITC) was developed which could be controlled by the intelligent mobile phone through Bluetooth. FITC is a popular fluorescent dye in biological application. According to its fluorescent characteristic, the light emitting diode (LED) with the wavelength of $490 \mathrm{~nm}$ was used as the elicitation light source. The photo-diode with the wavelength between 400 and $800 \mathrm{~nm}$ was used as fluorescence detector. They were integrated in an intelligent mobile phone controlled system. Experiments for FITC detection were executed based on the presented FITC detection system. At the lowest driving voltage ( $2.8 \mathrm{~V})$ for the exciting light source, the sensitivity was about $17 \mathrm{mV}$ per $\mathrm{mg} / \mathrm{ml}(\mathrm{R}=0.9956)$ in the rang of $0.345 \sim 5.52 \mathrm{mg} / \mathrm{ml}$.
\end{abstract}

\section{Introduction}

Florescence isothiocyanate (FITC) is a popular fluorescent dye, has been widely used in the for biological detection.[1,2,3] With the development of molecular biology and its integration with modern micro-machining technologies (or the so-called MEMS), lots of microchips based on FITC indicating strategy. [4,5,6] The combination of FITC labeled biological detection and MEMS promoted its application in the practical areas, such as pharmacology[7], food safety [8], cytology $[9,10]$, etc..

The success of FITC in both scientific researches and application developments activated lots of effort being invested into the construction of rapid and accurate FITC detection system. The classical FITC detection methods are visual observation, difference method, thin layer chromatography, high performance liquid chromatography tandem mass spectrometry and fluorescence spectrometer method. In these methods, the spectrometric methods have win the approval of users, but the equipment for the detection of FITC spectrum are cumbersome, so the FITC detection tests are restricted in the laboratories.

Because the traditional FITC detecting methods and equipment can not meet the demand of field test, portable and the ability of being incorporated with internet technology. A novel FITC detection system is proposed in this work, which can be controlled wirelessly by mobile phones (MP) with the Android system. According to FITC's fluorescent characteristic, the light emitting diode (LED) with the wavelength of $490 \mathrm{~nm}$ was used as the exciting light source. The photo diode with the wavelength between 400 and $800 \mathrm{~nm}$ was used as fluorescence detector. The functions of fluorescent excitation, detection and data storage are executed by the FITC detecting terminal, and the wireless controlling from the customers' MP is realized through Bluetooth.

\section{Materials and Methods}

Chemicals. FITC solutions are prepared according to the following steps: 1) $0.276 \mathrm{~g}$ FITC ( analytic degree) is dissolved in $25 \mathrm{ml}$ absolute ethyl alcohol and shaked well to get $11.04 \mathrm{mg} / \mathrm{ml}$ FITC stock solutions; 2) the $\mathrm{pH}$ value of the FITC stock solutions is adjusted to about 7 by the use of hydrochloric acid and sodium hydroxide; 3 ) the stock solutions are diluted to get the tested reagents which are the 5.52, 2.76, 1.38, 0.69 and $0.345 \mathrm{~g} / \mathrm{L}$. 
Methods for FITC detection. FITC is one of the fluorescent materials. When it is illuminated by the light with specific wavelength, they will absorb the energies of the photons. The absorbed energies can cause the electron transition from the ground state to the excited state. According to equation (1):

$$
E_{1}-E_{0}=h v_{1}=h \frac{\mathrm{c}}{\lambda_{1}}
$$

In which $E_{0}$ is the energy level of the ground state, $E_{l}$ is the energy level of the excited state, $h$ is Plank constant, $v_{l}$ is the frequency of the excitation light, $\lambda_{l}$ is the wavelength of the excitation light, $\mathrm{c}$ is the velocity of light in vacuum.

The excited state is the unstable state, the electrons in excited states will jump to the lower state $\left(E_{2}\right)$ and the energy they possessed will be emitted in the form of fluorescence with the wavelength $\lambda_{2}$. This process is described in the equation (2):

$$
E_{1}-E_{2}=h \frac{\mathrm{c}}{\lambda_{2}}
$$

The absorption and emission spectrum of FITC are given in Fig. 1. According to its absorption characteristic, when the wavelength of excitation light is around $500 \mathrm{~nm}$, its energy can be absorbed by FITC and the cause the energy transimision. So, the light emitting diode (LED) with the wavelength of $490 \mathrm{~nm}$ was used as the elicitation light source. Meanwhile, the wavelength of the emitted fluorescence is focused on a narrow region between 510 and $550 \mathrm{~nm}$, as given in the right curve of Fig.1. So the photo-diode with the wavelength between 400 and $800 \mathrm{~nm}$ is selected as the detector for emitted fluorescence.

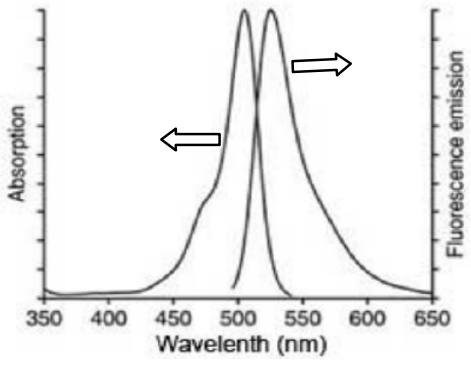

(A)

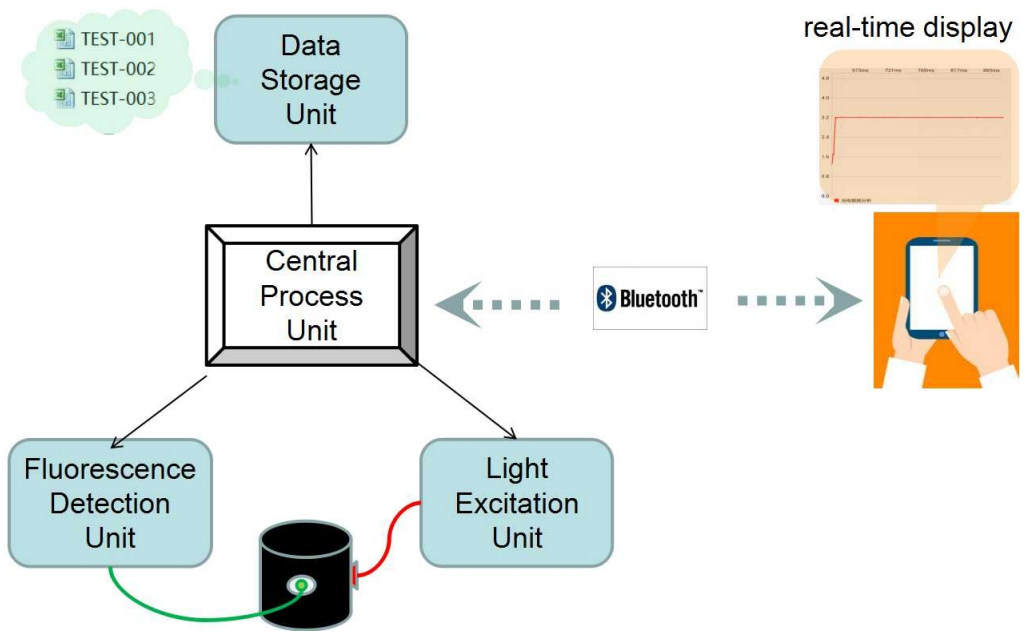

(B)

Fig. 1. The absorption (left curve) and emission (right curve) spectrum of FITC (A) and construction for the MP controlled FITC detection system (B)

System design. The construction of the MP controlled FITC detection system is outlined in Fig.1 (B). The user's commands are send from MP to the central process unit (CPU) through Bluetooth. Then these commands are distributed to the light excitation unit and the fluorescence detection unit in order to realize the FITC detection. The detected fluorescent information is measured as the output voltage of fluorescence detector, named as $\mathrm{V}_{\text {detect }}$. The data of $\mathrm{V}_{\text {detect }}$ is saved as an excel file in the SD card, and a real-time and dynamic curves of this voltage signal is displayed on the screen of MP.

Optical parts. The package of the chamber for holding the tested sample is in the form of Blackbody to guarantee there is no access for environmental light entering. Moreover the inlet for the excitation illumination and the outlet for fluorescence detection are designed as shown in Fig. 1(B). They are located at the edge of the package in the same horizontal section and the angle of these two poles with the center chamber is 90 degree. This design is to avoid the interference of the excitation light. 
Excitation light unit. The excitation light source is HSE490H-M506. Its driving power is from the voltage stabilizing chip. The circuit of this unit is presented in Fig. 2 (A) in which the device $\mathrm{D}_{2}$ is HSE490H-M506. The voltage applied on $\mathrm{D}_{2}$ can be adjusted by altering the value of the resistor $\mathrm{R}_{2}$. In this work, the voltages of $\mathrm{D}_{2}$ are controlled at 2.8, 2.9, 3.0 and $3.1 \mathrm{~V}$, respectively.
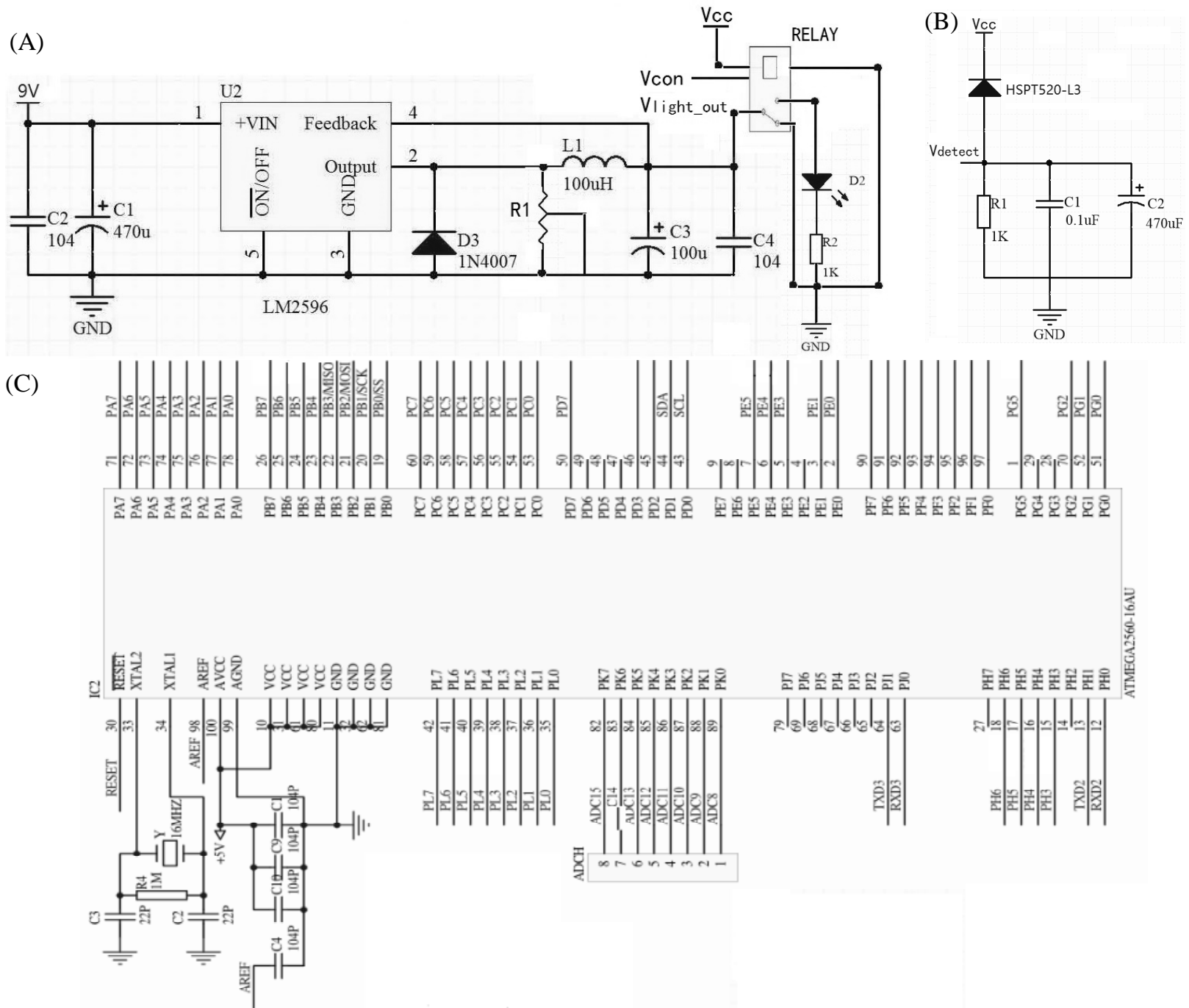

Fig. 2. The circuits for the ecitation light unit (A), the fluorescece detection unit (B) and the central processing unit (C).

Fluorescence detection unit. The photo-diode HSPT520-L3 is used as fluorescence detector. Its typical driving circuit is adorpted as presented in Fig. 2 (B), in which $\mathrm{V}_{\text {detect }}$ is the fluorescence sensing signal. When there is no fluorescence in the chamber, the detecor HSPT520-L3 is in the state of high resistor, $\mathrm{V}_{\text {detect }}$ is low. On the contrary, when there is fluorescence, its resistor is reduced, so $\mathrm{V}_{\text {detect }}$ is increased.

Central processing unit. The chip Atmega 2560 is used as kernal device of the central processing unit (CPU). The typical circuit of CPU is presented in Fig. 2 (C).

\section{Results and Discussion}

The proposed FITC detection system is examined by measuring different concentrations FITC solutions, the results are presented in Fig. 3. 


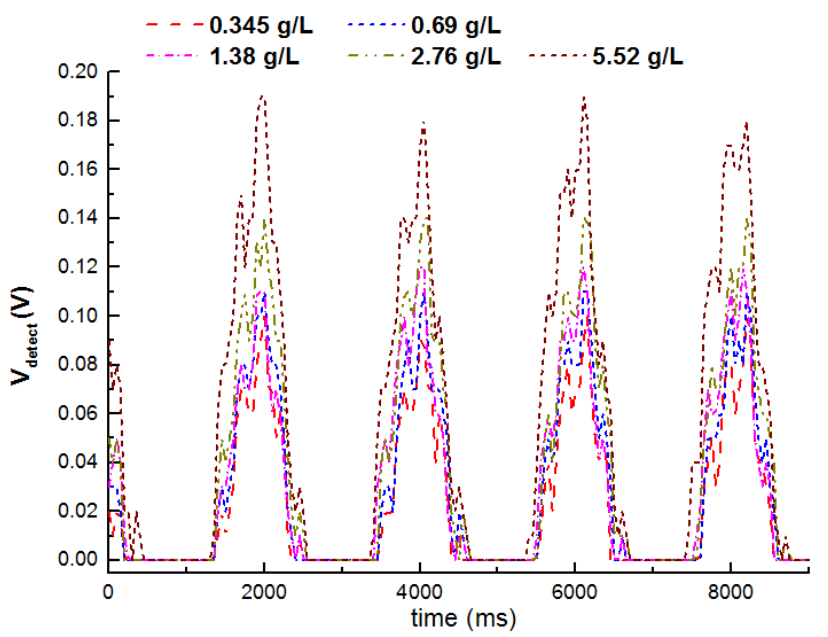

(A)

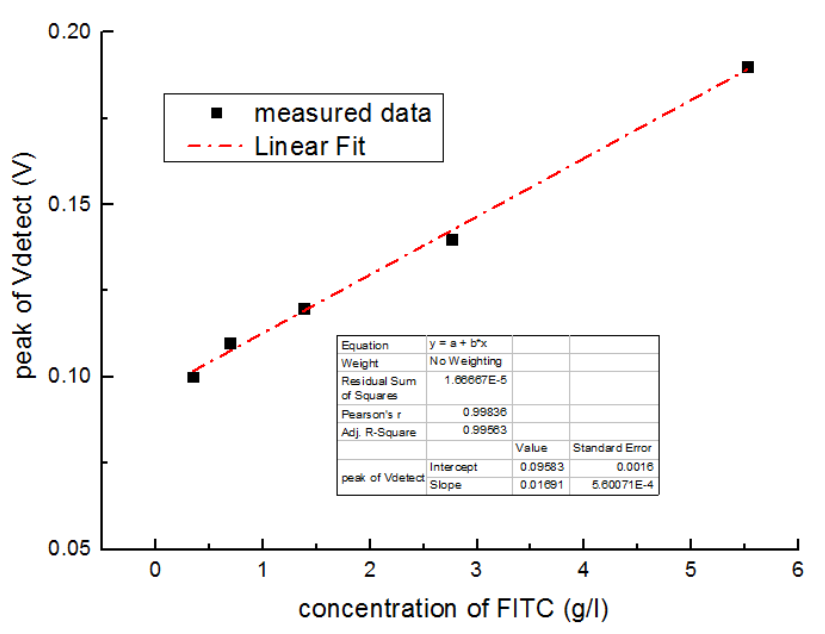

(B)

Fig. 3. The detection results for different concentrations of FITC. (A) is detected instantaneous signal and (B) is the peak of the deteced output voltage in FITC solutions with different concentrations.

\section{Conclusions}

The proposed MP controlled FITC detecting system is verified in the experiments for different concentrations of FITC. It is believed that the presented MP controlled FITC detection system is a potential model for other kinds of fluorescence materials by changing the exciting light source. Furthermore, in the presented FITC detecting model, MP's screen and Bluetooth are utilized as the users' interface for displaying detection results instantly and wireless communication block, respectively, so the cost, the weight and volume in the presented work are lowered, while the functions are increased with the potential application for the so-called Internet Plus technologies.

\section{Acknowledgements}

This work was financially supported by the National Natural Science Foundation of China (61371028) and Tianjin Natural Science Foundation (15JCTPJC63900, 15ZCZDSF00060).

\section{References}

[1] P. Balogh, G. Szekeres and P. Németh: Journal of Immunological Methods, Vol.169 (1994) p. 35.

[2] I.J. Harmer and D. Samuel: Journal of Immunological Methods, Vol. 122 (1989) p. 115.

[3] V.S. Trubetskoy, N.V. Koshkina, V.G. Omel'yanenko, V.L. L'vov, B.A. Dmitriev, A.B. Petrov and V.P. Torchilin: FEBS Letters, Vol. 269 (1990), p.79.

[4] K. K.R. Tetal and M.A. Vijayalakshmi: Analytica Chimica Acta, Vol. 906 (2016) p. 7-21.

[5] X. Chen, D.F. Cui and L. L. Zhang: Nanotechnology and Precision Engineering, Vol. 7(2) (2009) p. 127

[6] D. S. Li, Y. Xue, J.L. Peng, Z. Zhou, J. Gan and Y. Wang: Chem. J. Chinese Universities, Vol.33 (2012) p. 49

[6] M. Korampally, S.J. Apperson, C.S. Staley, J. A. Castorena, R. Thiruvengadathan, K. Gangopadhyay, R. R. Mohan, A. Ghosh, L. Polo-Parada and S. Gangopadhyay: Sensors and Actuators B: Chemical, Vol. 171-172 (2012) p. 1292.

[7] J. Yang, J. Yao, J. Chen, X.N. Wang, T.Y. Zhu, L.L. Chen and P. Chu: European Journal of Pharmacology, Vol. 618 (2009) p. 1. 
[8] C. Song, J. Liu, J. Li and Q. Liu: Biosensors and Bioelectronics, Vol. 85 (2016) p. 734.

[9] Y.W. Wu, S. F. Chen, C. B. Yang and Y. H. Tsai: Journal of Chromatography B, Vol. 863 (2008) p.187.

[10] R. Yumoto, S. Suzuka, K. Oda, J. Nagai and M. Takano: Drug Metabolism and Pharmacokinetics, Vol. 27 (2012) p. 336 